\title{
Overactive Bladder Disease: The Urge for Better Therapies
}

\author{
Lisa Thomas, PharmD, and Eric J. Culley, PharmD
}

$\mathrm{I}$

n the April 2008 issue of JMCP, D'Souza et al. compared persistence, adherence, and switch rates for immediate-release (IR) and extended-release (ER) formulations of oxybutynin and tolterodine for patients enrolled in a regional managed care health plan. Only $55.5 \%$ of patients refilled their first prescription for an overactive bladder (OAB) medication, and 13.2\% persisted with the index medication for 1 year. The switch rate was $13.3 \%$ for the overall sample and $24.0 \%$ of patients with at least 1 refill. Although no difference was observed for persistence rates for any of the $4 \mathrm{OAB}$ drugs, adherence rates were slightly higher for ER versus IR drugs. ${ }^{1}$ We commend D'Souza and colleagues for their efforts in the development of these findings. However, the results of this analysis are confounded by 2 major factors: (1) several limitations of retrospective data analyses and (2) the challenges associated with the clinical management of OAB. Also, the slight advantage offered by ER drugs raises a potential question regarding the "true" value offered by once-daily dose regimens versus multiple-dose regimens.

In the category of difficulty in clinical management, low persistence and adherence rates associated with $\mathrm{OAB}$ therapies may be attributable to the undesirable adverse events associated with anti-cholinergic agents, including dry mouth, constipation, tachycardia, and blurred vision. ${ }^{2}$ Some postulate that the ER drug formulations could minimize the undesirable events and increase patient compliance by maintaining flat plasma drug concentrations. ${ }^{3,4}$ However, available data suggest that less frequent dosing intervals do not necessarily translate to increased compliance, indicating that side effects and inconvenience alone cannot explain non-adherence.

Medication adherence and persistence are also dependent on the perceived benefit of therapy experienced by the patient. Humanistic outcomes, such as quality of life, offer a greater understanding of the potential benefits offered by various drug therapies but are just starting to be included in clinical trials. This lack of quality-of-life data is an important deficit in currently available information that needs to be addressed since the statistically significant effects observed for anti-muscarinic drugs in clinical trials (e.g., urinary frequency, volume voided per micturition) may not always translate to a clinically relevant benefit for patients in the real world.

\section{Limitations of Retrospective Claims Analyses}

The analysis by D'Souza et al., like other administrative claims analyses of $\mathrm{OAB}$, provides a glimpse of the challenges faced both by researchers and by clinicians in the effort to identify improved strategies to diagnose and manage OAB. ${ }^{1}$ Various adherence and persistence studies pertaining to $\mathrm{OAB}$ and anti-muscarinic agents have been published. In long-term, open-label studies, the percentages of patients who persisted on therapy for approximately 10 to 12 months were 46\%,76\%, 81\%, and $81 \%$ for oxybutynin ER, tolterodine ER, trospium, and solfenacin, respectively. ${ }^{5-8}$ It should be recognized, however, that the observed persistence rates in controlled trials might not be reflective of real-world clinical settings. Furthermore, there are few published studies on the topic of long-term persistence with drug therapy among $\mathrm{OAB}$ patients in the United States within a usual-care setting. For example, a 1995-1996 study of treatment of urge incontinence in U.S. veterans (receiving primarily oxybutynin chloride [39.8\%], dicyclomine hydrochloride [16.0\%], or imipramine hydrochloride [13.9\%]) demonstrated that fewer than $30 \%$ of patients continued on therapy for 1 year. ${ }^{9}$

Although findings of D'Souza et al.'s database analysis and other similar studies highlight differences between real-world and clinical trial results, among the most pressing challenges in research on $\mathrm{OAB}$ is the question of whether samples used in most database studies adequately represent the population of patients with the disorder. The prevalence of $\mathrm{OAB}$ increases markedly with advancing age in both men and women. ${ }^{10}$ A populationbased U.S. study of women enrolled in a large health maintenance organization suggests a rate of urinary incontinence greater than $50 \%$ among women aged 60 to 90 years. ${ }^{11}$ Unfortunately, data relating to the prevalence and impact of $\mathrm{OAB}$ are sparse for this subset of patients. Most OAB studies, including the current analysis published by D'Souza et al., include patients aged 18 years or older, with the mean age of patients ranging from 56 to $61 .^{12-14}$ Therefore, it is difficult to assess how the truly older population with $\mathrm{OAB}$ responds to antimuscarinic therapy. Anecdotally, there is a belief that antimuscarinic agents are often underutilized in the elderly despite the marked increase in the prevalence of $\mathrm{OAB}$. This alleged underuse may result from concerns about the frequency of more serious anti-cholinergic adverse events, such as cognitive impairment and sleep disturbances, as well as drugdrug interactions with existing pharmacotherapy. ${ }^{12}$

Additionally, studies conducted with commercial databases, like the one used by D'Souza, may not adequately represent the populations in which research is most urgently needed. For example, although not included in D'Souza et al.'s analysis, Medicaid enrollees represent an important potential target of better treatment interventions for OAB. Both the proportion of women and per-capita health care expenditures are higher for adults with Medicaid coverage than adults with other types of insurance coverage. ${ }^{15}$ Contributing factors to poor compliance include low level of education, cultural and social support factors, and adverse drug reactions. ${ }^{16,17}$ These factors may be more prevalent among underserved populations such as Medicaid beneficiaries. Therefore, a higher prevalence of $\mathrm{OAB}$ and lower 
persistence rates are more likely to exist in the Medicaid population as compared with commercially insured plans. ${ }^{18}$

One study that addressed the need for research on OAB in Medicaid populations was reported by Yu et al. in $2005 .{ }^{19} \mathrm{Yu}$ et al. studied persistence (discontinuation of OAB drug therapy and time to discontinuation) and adherence (medication possession ratio [MPR]) in a Medicaid population with chronic $\mathrm{OAB}$ or urinary incontinence from January 1999 to April 2002. The average age of $\mathrm{OAB}$ patients at the time of index pharmacy claim was 63.2 years $(S D=16.1)$, slightly higher than the mean age reported in the analysis by D'Souza et al. $(55.7, \mathrm{SD}=14.5)$. Most Medicaid patients in the study by Yu et al. began treatment with tolterodine or oxybutynin. Of the 2,496 eligible patients that were included in the Yu et al. study, 36.9\% had no refill after the index pharmacy claim. During the 6-month follow-up period, two thirds (66.9\%) of patients did not remain on their treatment regimen for more than 90 days, and $77.7 \%$ of patients did not remain on their treatment for longer than 150 days. The median time to discontinuation was 50 days. On average, about one third of the follow-up period was covered by $\mathrm{OAB}$ medications, only $4.8 \%$ of patients renewed their prescriptions within a 30-day gap allotted for refills, and $50 \%$ of patients received pharmacological treatment for less than 60 days cumulatively during the 6-month follow-up period. Only 122 patients (4.9\%) exhibited $>80 \%$ MPR in the initial 6-month follow-up period, During the additional 6-month follow-up period, the rate of discontinuation increased to $88.6 \%$, the mean MPR dropped to 0.22 , and the adherence rate, measured as MPR $>0.80$, was a mere $0.7 \% .{ }^{19}$

Despite different populations, $100 \%$ Medicaid in Yu et al. versus 58\% commercially insured, 19\% Medicare, and 23\% selfinsured employers in D'Souza et al., their findings regarding low persistence and medication adherence are similar. D'Souza found that only $55.5 \%$ of patients refilled their index $\mathrm{OAB}$ medication (they did not report continuation on any $\mathrm{OAB}$ drug for at least 1 refill but did report $80 \%$ discontinuation of all OAB medications at 6 months follow-up) compared with $63.1 \%$ of patients who had at least 1 refill in the Medicaid population studied by Yu et al. D'Souza et al. found a median 31 days to discontinuation of the index OAB drug therapy versus 50 days in Yu et al.,19

However, a comparison of the Yu et al. and D'Souza et al. reports provides a reminder of the need to consider the appropriateness of study methodologies in interpreting study findings. For example, although the gap between prescription fills was used to determine discontinuation rates in both analyses, D'Souza used a medication-uncovered interval of 45 days to define discontinuation versus $>30$ days in the study Yu et al. The length of this gap varies between studies; 45 days has been used prior to D'Souza et al.'s analysis.9,20 Evidence to suggest which gap length is most appropriate to assess persistence among OAB patients or why patients may even have a gap in therapy is lacking, suggesting a need to reevaluate this potentially important methodological decision. ${ }^{19}$
Similarly, evaluation of the methods and outcome measures in the 2 studies by Yu et al. and D'Souza et al. precipitates consideration of the appropriate measure of medication adherence for OAB. Both studies used the common MPR benchmark of $\geq 0.80$ to define adherence. Yu et al., in fact, suggest that the nature of clinical differences among various therapeutic classes may warrant the use of different definitions of adherence for drugs used for different diseases or conditions; in other words, use of the same definition of adherence may result in overestimation or underestimation of true compliance among drug classes. Therefore, there is a need for further research to develop disease-specific MPR threshold values to achieve a more accurate estimation of clinically meaningful adherence rates. ${ }^{19}$

In comparing the results reported by D'Souza et al. and Yu et al., it is also necessary to consider how different methods to select patients may affect study findings. Yu et al. identified patients with a pharmacy claim for at least 1 OAB medication in addition to either (a) 2 or more confirmed diagnoses during the study period or (b) at least 1 diagnosis of OAB made at any time after the index date. Yu et al. did not report the proportion of Medicaid patients who received an OAB drug but did not meet either of the second criteria for inclusion in the study, while D'Souza et al. found that only 53.7\% $(n=600)$ of 1,117 patients who received OAB drug therapy were diagnosed with at least 1 symptom characteristic of OAB based on International Classification of Diseases, Ninth Revision, Clinical Modification codes during the 18-month eligibility period. The additional criteria for selection in the analysis by Yu et al. may yield a higher degree of confidence that the patients had chronic OAB than in the D'Souza analysis.

Another potential issue with D'Souza et al.'s methodology is the definition of persistence used. For any given chronic disease state, a switch in therapy is neither uncommon nor inappropriate. Due to the tolerability profile of anti-muscarinic agents, it is likely that patients switch to another dose of the same drug or are switched to an entirely different drug. Hence, in the analysis by Yu et al., a patient making a switch from one brand of OAB drug to another was considered to be persistent with therapy since the patient was considered to still be receiving active therapy. In contrast, D'Souza et al.'s definition of non-persistence included a switch to a different $O A B$ medication or even to a different dosage form of the same drug. This analysis also failed to look past a first-time switch to determine if patients remained on the second treatment.

Failure to consider the possible role that other medication classes play in producing the observed outcomes represents another limitation of the analysis by D'Souza et al. Of growing concern among geriatricians (and others) is the potential for adverse events of drug treatment for 1 condition that affects other coexisting conditions. ${ }^{21}$ Polypharmacy and the chronic use of multiple drugs are realities for many elderly patients. ${ }^{22}$ Depression and anxiety are common comorbidities among $\mathrm{OAB}$ 
patients. ${ }^{2,23}$ Approximately 17\% of patients in D'Souza et al.'s sample were diagnosed with comorbid depression or anxiety, yet they failed to examine concomitant drug utilization that could potentially cause anticholinergic toxicity. ${ }^{1}$ This limitation may have significantly confounded the study's reported rates of adherence and persistence.

Pharmacy benefit design can also influence medication adherence and persistence and is often overlooked in studies using large databases of administrative claims that are compiled from multiple employers, health plans, and payer types; the effects of benefit design were also unstudied in the D'Souza et al. analysis. Employers and health plans offering drug benefits employ a variety of management tools to manage utilization and costs. Many of these tools are centered on the use of a drug formulary, prior authorization, quantity level limits, and mail-order pharmacies. Mail-order pharmacies typically dispense a 90-day supply of medication during a single fill, as well as provide automatic refills for most drugs. These policies can inflate measures of adherence as measured by the days supply of drug received. D'Souza et al. failed to provide an assessment of the drug groups' comparability with respect to formulary status, tiering, benefit design, and management of edits (e.g., step therapy, prior authorization), which could possibly have confounded persistence, adherence, and switch rates within this analysis. Furthermore, this analysis failed to address the impact of the exclusion of cash-paying customers on a study drug during the time frame of this analysis. Lastly, no information specific to mail-order use in this population was addressed, although any mail-order use could have significantly impacted the results of this analysis.

\section{The Importance of Patient-Reported Outcomes in OAB}

The work of D'Souza et al. and similar studies, suggest areas for improvement in future research. OAB disease is a prevalent condition that has a negative impact on an individual's healthrelated quality of life (HRQL). ${ }^{23,24}$ Although the efficacy of antimuscarinic agents has been demonstrated in various clinical trials, information pertaining to the clinical significance of these data are a more recent addition to the literature. Data to support patient satisfaction with the purported benefits of drug therapy is essential in order to determine the true strengths and limitations of this class of medications.

Traditionally, clinicians have greatly relied on objective measures, such as urodynamic studies (series of office tests to observe the function of the lower urinary tract) for evaluating the efficacy of treatment. Objective measures can help facilitate the diagnosis and management of $\mathrm{OAB}$, but because $\mathrm{OAB}$ is a symptom-based syndrome, its management is highly contingent upon a patient's report of symptoms. Patient-reported outcomes, assessed by subjective measures, provide an opportunity to improve one's understanding of $\mathrm{OAB}$ outcomes and may even help to redefine a treatment success. ${ }^{25}$ As stated by Fairclough et al., "While we can measure a biological response, we may not be able to determine whether that response makes a noticeable difference to the patient." 26

Treatment with anti-muscarinic agents in the management of $\mathrm{OAB}$ typically requires at least 4 to 8 weeks of continuous therapy before the benefits of therapy are realized. There is also evidence to suggest that $\mathrm{OAB}$ patients who continue their medication have greater improvement in relevant symptoms and utilize fewer resources. ${ }^{27}$ Few studies have assessed the clinical benefit of persistence with OAB/urinary incontinence (UI) drug therapy, but the logistic regression analysis performed by Yu et al. suggests that the risk of urinary tract infections is 37\% (odds ratio $[\mathrm{OR}]=1.37,95 \%$ confidence interval $[\mathrm{CI}]=1.03-1.84$, $P=0.03$ ) higher among those who discontinue OAB therapy. ${ }^{19}$ D'Souza et al.'s work would have provided more insight regarding the management of OAB if patient-reported outcomes (PROs) had been included as end points, but this is a limitation of administrative claims data in which clinical information is not captured.

Incorporating patient-reported outcomes into clinical trials has more recently become a common means of assessing treatment efficacy for symptom-based conditions. Studies that include assessments of both subjective and objective outcomes provide results that represent a more global patient experience compared with those captured by evaluating either type of measure alone. In some studies, objective end points demonstrated larger differences between treatment groups compared with subjective measures. ${ }^{25}$ For example, a trial of oxybutynin IR in elderly patients demonstrated that patients receiving oxybutynin IR experienced statistically significant reductions in daytime micturitions during a 2-week period compared with those receiving placebo. After 6 weeks of treatment, the percentage of patients who perceived a benefit of treatment appeared to be greater in the treatment group compared with placebo group (79\% vs. 55\%), but the difference was not statistically significant $(P=0.09)$. Furthermore, patients were asked to grade their improvement based on a 4-point ranked ordinal scale; ratings failed to demonstrate significant differences between groups at the end of the study. ${ }^{25,28}$

In a placebo-controlled trial of tolterodine IR and oxybutynin IR, 12 weeks of treatment with tolterodine IR significantly reduced 24-hour micturitions compared with placebo. Patients receiving oxybutynin IR demonstrated statistically significantly reduced 24-hour urge UI episodes compared with placebo. Both active treatments increased volume voided per micturition. Despite the favorable effects of tolterodine IR and oxybutynin IR on bladder diary end points, the percentages of patients perceiving improvement in bladder condition after 12 weeks of treatment were comparable for placebo, tolterodine IR, and oxybutynin IR at rates of $47 \%, 50 \%$, and $49 \%$, respectively. ${ }^{29}$

Objective measures are important in the assessment and management of patients, but they are probably not valid indicators of the disease burden experienced by patients with OAB. 
Despite the importance of including subjective measures in the management of $\mathrm{OAB}$, few studies include subjective measures as a primary end point. Future studies with a greater emphasis on subjective measures using instruments that have undergone validation studies, such as $\mathrm{OAB}$ questionnaires, are needed in order to gain a greater understanding of $\mathrm{OAB}$ as a chronic disease. ${ }^{25}$

\section{Defining an Optimal Dosing Regimen for Medication Compliance}

In a real-world setting, achieving adherence to prescribed medication is challenging. Specific disease characteristics associated with various chronic disease states may have a significant impact on adherence. ${ }^{30}$ For example, asymptomatic diseases, such as hypertension and hyperlipidemia are commonly associated with poor compliance and adherence. ${ }^{31,32}$ A prospective study examining adherence to the Joint National Committee on Hypertension (JNC VI) treatment guidelines in the New York metropolitan area demonstrated that only $37 \%$ of the 821 patients surveyed reported consistent adherence to their anti-hypertensive regimen. ${ }^{33}$ In a 1982 study of a cross-sectional sample of 800 adults in Detroit, Michigan, 21\% of the 206 hypertensive patients interviewed had stopped drug treatment without being instructed to do so. ${ }^{34}$ Patient perception of health status was the most common factor found to distinguish dropouts from those adherent to their medication regimens; poorer perceived health was associated with higher rates of adherence to the prescribed treatment regimen. Patients who felt well without medication were more likely to discontinue their medications on their own. Therefore, the clinical efficacy rates demonstrated by numerous "blockbuster" medications in controlled clinical trials frequently do not correlate with outcomes observed in real-world settings. The implication for real-world results is that large reductions in morbidity and/or mortality seen in clinical trials may not always translate into a better prognosis for patients. ${ }^{30}$

ER formulations of many drugs have been developed, in theory, to offer increased patient compliance versus multiple daily-dosing regimens by reducing tablet burden. Although limited information is available within the literature specific to compliance patterns of ER drug formulations in $\mathrm{OAB}$, data extrapolated from studies specific to osteoporosis and epilepsy suggest that less frequent dosing intervals do not guarantee increased medication compliance and may actually compromise therapeutic efficacy. For example, compliance and persistence studies conducted in patients with osteoporosis have demonstrated a limited benefit of bisphosphonates dosed monthly or even weekly. One-year persistence rates across several analyses ranged from $18.5 \%$ to $55.7 \%$ for the daily-dosed regimen versus $22.1 \%$ to $69.7 \%$ for the weekly-dosed regimen. ${ }^{35}$ Furthermore, Weiss et al. determined that, on average, persistence with monthly dosed ibandronate (98 days) was significantly lower than with weekly alendronate (116 days) or weekly risedronate (113 days) $(P<0.001){ }^{35}$
In disease states such as epilepsy, it is questionable whether flat plasma concentrations offered by ER drug formulations of anti-epileptic drugs (AEDs) improve anti-epileptic efficacy compared with fluctuating plasma concentrations. It is more certain that they minimize concentration-related adverse effects. ${ }^{3,4}$ From a patient compliance perspective, it is unknown whether once-daily administration of AEDs is "better" than twice-daily administration. Levy et al. demonstrated that switching dosing schedules from multiple doses per day to once daily by the use of ER drug formulations did not generally improve therapeutic coverage..$^{36}$ Also, from a pharmacokinetic perspective, the impact of a missed dose is greater with large doses and less frequent administrations. ${ }^{37}$ Therefore, the potential risk of breakthrough seizure is greater during AED once-daily administration versus twice-daily administration. Consequently, the potential for increased compliance should be weighed against the real impact of a missed dose. In general, from a (theoretical) pharmacokinetic perspective, unless the incidence of non-compliance is reduced by greater than two thirds when a medication is normally taken once daily rather than 3 times daily, the increased compliance offered by the reduction in dose administration frequency is likely to be of no advantage and can be counterproductive in minimizing the occurrence of subtherapeutic drug concentrations. ${ }^{36}$ In essence, as stated by Urquhart et al., focusing only on dosing frequency can lead to false conclusions about therapeutic superiority, with the crucial matter being not the percentage of prescribed doses taken, but rather the continuity of therapeutic action. $^{38}$

Despite advances in our understanding of the pathophysiology of the lower urinary tract, muscarinic receptor antagonism remains to be the only clinically proven mechanism of relieving the symptoms of $\mathrm{OAB}$. OAB patients who discontinue treatment are unable to obtain the full benefit of therapy. Establishing effective intervention programs to improve patient persistence and adherence would seem to be a worthwhile initiative with respect to improving health outcomes. While patient education is an important factor, improved patient monitoring by both physicians and pharmacists to identify noncompliant populations is equally important. It would be useful to see the results of a study assessing the effect of upfront counseling on a patient's attitudes, realistic treatment goals, and compliance barriers. Although the therapeutic armamentarium for $\mathrm{OAB}$ has expanded, the discontinuation rates of the antimuscarinic agents observed in clinical trials remain a common shortcoming. This suggests that there is still an unmet need for agents with superior clinical effectiveness and tolerability profiles that can provide increased rates of adher-

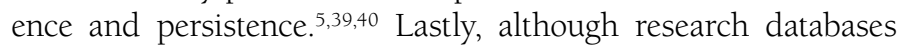
contain useful information pertaining to real-world adherence and persistence patterns, several assumptions pertaining to patient-reported outcomes, benefit designs, and other confounding factors must be made when interpreting such results. Until more effort is made to incorporate such information into these 
types of analyses, the clinical and practical utility of results obtained from research databases remains questionable.

\section{Authors}

LISA THOMAS, PharmD, is a clinical pharmacy services specialist, and ERIC J. CULLEY, PharmD, is the clinical pharmacy services manager at Highmark Blue Shield, Pittsburgh, Pennsylvania. Culley is a member of the JMCP Editorial Advisory Board.

AUTHOR CORRESPONDENCE: Lisa Thomas, PharmD, Clinical Pharmacy Services Specialist, Highmark Blue Shield, 120 Fifth Ave., Suite 1812, Pittsburgh, PA 15222. Tel.: 412.544.5075;

Fax: 412.544.4527; E-mail: lisa.thomas@highmark.com

\section{DISCLOSURES}

The authors report no conflicts of interest related to the subject of this commentary.

\section{REFERENCES}

1. D'Souza AO, Smith MJ, Miller LA, et al. Persistence, adherence, and switch rates among extended-release and immediate-release overactive bladder medications in a regional managed care plan. J Manag Care Pharm. 2008;14(3):291-301. Available at: www.amcp.org/data/jmcp/JMCPMaga_ April08_291-301.pdf.

2. Ouslander JG. Management of overactive bladder. N Engl J Med. 2004;350:786-99.

3. Bialer M. Pharmacokinetic evaluation of sustained release formulations of antiepileptic drugs. Clin Pharmacokinet. 1992;22:11-21.

4. Bialer M, Cloyd JC. General principles: formulations and routes of administration. In: Levy RH, Mattson RH, Meldrum BS, eds. Antiepileptic Drugs. 4th ed. New York; Raven Press; 1995:161-78.

5. Halaska M, Ralph G, Wiedemann A, et al. Controlled, double-blind, multicentre clinical trial to investigate long-term tolerability and efficacy of trospium chloride in patients with detrusor instability. World J Urol. 2003;20:392-99.

6. Diokno A, Sand P, Labasky R, et al. Long-term safety of extended-release oxybutynin chloride in a community-dwelling population of participants with overactive bladder: a one-year study. Int Urol Nephrol. 2002;34:43-49.

7. Haab F, Cardozo L, Chapple C, et al. Long-term open-label solfenacin treatment associated with persistence with therapy in patients with overactive bladder syndrome. Eur Urol. 47:376-84.

8. Kreder K, Mayne C, Jonas U. Long-term safety, tolerability and efficacy of extended-release tolterodine in the treatment of overactive bladder. Eur Urol. 2002;41:588-95.

9. Malone DC, Okano GJ. Treatment of urge incontinence in veterans affairs medical centers. Clin Ther. 1999;21:867-77.

10. Stewart WF, Van Rooyen JB, Cundiff GW, et al. Prevalence and burden of overactive bladder in the United States. World J Urol. 2003;20:327-36.

11. Melville JL, Katon W, Delaney K, et al. Urinary incontinence in U.S. women: a population-based study. Arch Intern Med. 2005;165:537-542.

12. Staskin D. Overactive bladder in the elderly: a guide to pharmacological management. Drugs Aging. 2005;22(12):1013-28.

13. Rudy D, Cline K, Harris R, et al. Multicenter phase III trial studying trospium chloride in patients with overactive bladder. Urology. 67:275-80.

14. Roberts R, Bavendam T, Glasser B, et al. Tolterodine extended release improves patient reported outcomes in overactive bladder: results from the IMPACT trial. Int J Clin Pract. 2006;60:752-58.
15. Kaiser Commission on Medicaid and the Uninsured. Medicaid:a lower cost approach to serving a high-cost population. Available at: www.kff.org/ medicaid/7057a.cfm. Accessed on April 1, 2008.

16. Shea S, Misra D, Ehrlich M, et al. Correlates of nonadherence to hypertension treatment in an inner city minority population. Am J Public Health. 1992;82:1607-12.

17. Kreuger KP, Felkey BG, Berger BA. Improving adherence and persistence: a review and assessment of interventions and description of steps toward a national adherence initiative. J Am Pharm Assoc. 2003;43:668-78.

18. Shaya F, Blume S, Gu A, Zyczynski T, et al. Persistence with overactive bladder pharmacotherapy in a medicaid population. Am J Manag Care. 2005;11(4 suppl):S121-S129.

19. Yu YF, Nichol MB, Yu AP, Ahn J. Persistence and adherence of medications for chronic overactive bladder/urinary incontinence in the California Medicaid program. Value Health. 2005;8(4):495-505.

20. Avorn J, Monette J, Lacour A, et al. Persistence of use of lipid-lowering medications. A cross-national study. JAMA. 1998;279:1458-62.

21. Tinetti ME, Bogardus ST, Agostini JV. Potential pitfalls of disease specific guidelines for patients with multiple conditions. N Engl J Med. 2004; 351:2870-74

22. Erdem N, Chu F. Management of overactive bladder disease and urge urinary incontinence in the elderly patient. Am J Med. 2006;119:29-36.

23. Liberman JN, Hunt TL, Stewart WF, et al. Health related quality of life among adults with symptoms of overactive bladder: results from a U.S. community-based survey. Urology. 2001;57:1044-50.

24. Coyne K, Payne C, Bhattacharyya S, et al. The impact of urinary urgency and frequency on health related quality of life in overactive bladder: results from a national community survey. Value Health. 2004;7:451-59.

25. Abrams P, Artibani W, Jerzy B, et al. Assessment of treatment outcomes in patients with overactive bladder: Importance of objective and subjective measures. Urology. 2006;68(suppl 2A):17-28.

26. Fairclough DL. Patient reported outcomes as endpoints in medical research. Stat Methods Med Res. 2004;13:115-38.

27. Brown JS, McGhan WF, Chokroverty S. Comorbidities associated with overactive bladder. Am J Manag Care. 2000;6(11 suppl): S574-S579.

28. Szonyi G, Collas DM, Ding YY, et al. Oxybutynin with bladder training for detrusor instability in elderly people: a randomized controlled trial. Age Ageing. 1995;24:287-91.

29. Abrams P, Freeman R, Anderstorm C, et al. Tolterodine, a new antimuscarinic agent: as effective but better tolerated that oxybutynin in patients with an overactive bladder. Br J Urol. 1998;81:801-10.

30. Frishman W. Importance of medication adherence in cardiovascular disease and the value of once-daily treatment regimens. Cardiology. 2007; 15:257-62.

31. Osterberg L, Blaschke T. Adherence to medication. N Engl J Med. 2005; 353:487-97.

32. Jackevicius CA, Mamdani M, Tu JV. Adherence with statin therapy in elderly patients with and without acute coronary syndromes. JAMA. 2002; 288:462-67.

33. Chen JW, Kalis MM, Feifer S. Patient reported adherence to guidelines of the sixth joint national committee on prevention, detection, evaluation and treatment of high blood pressure. Pharmacotherapy. 2001;21:828-41.

34. Cummings KM, Kirscht JP, Binder LR, et al. Determinants of drug treatment maintenance among hypertensive persons in inner city Detroit. Public Health Rep. 1982;97:99-106.

35. Weiss T, Henderson S, McHorney C, et al. Persistence across weekly and monthly bisphosphonates: analysis of U.S. retail pharmacy prescription refills. Curr Med Res Opin. 2007;23:(9):2193-S203.

36. Levy G. A pharmacokinetic perspective of medicament noncompliance. Clin Pharmacol Ther. 1993;54:242-44. 
37. Rowland M, Tozer T. Clinical Pharmacokinetics: Concepts and applications. 3rd ed. Baltimore, MD: Williams \& Wilkins; 1995:83-103.

38. Urquhart J. Ascertaining how much compliance is enough with outpatient antibiotic regimens. Postgrad Med J. 1992;68(suppl 3):S49-S58.

39. Garley AD, Kaufman JM, Sand PK, et al. Symptom bother and health related quality of life outcomes following solifenacin treatment for overactive bladder: the VESICARE Open Label Trial (VOLT). Clin Ther. 2006; 28(11):1935-46.
40. Foote J, Glavin K, Kralidis G, et al. Treatment of overactive bladder in the older patient: pooled analysis of three phase III studies of darifenacin an M3 selective receptor antagonist. Eur Urol. 2005; 48(3):471-77. 